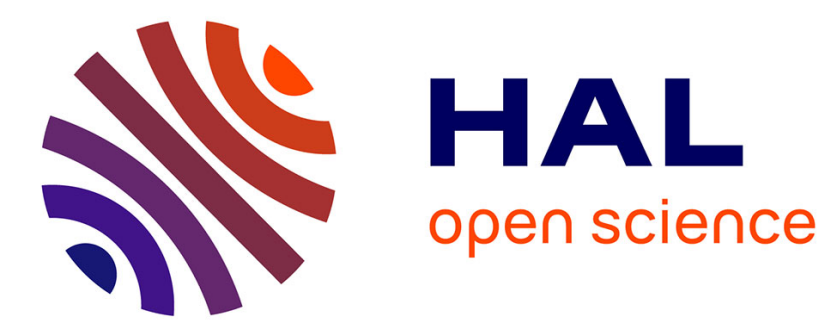

\title{
Efficient single-mode Brillouin fiber laser for low-noise optical carrier reduction of microwave signals
}

\author{
Stéphanie Norcia, Sylvie Tonda-Goldstein, Daniel Dolfi, Jean-Pierre
}

Huignard, Robert Frey

\section{To cite this version:}

Stéphanie Norcia, Sylvie Tonda-Goldstein, Daniel Dolfi, Jean-Pierre Huignard, Robert Frey. Efficient single-mode Brillouin fiber laser for low-noise optical carrier reduction of microwave signals. Optics Letters, 2003, 28 (20), pp.1888-1890. hal-00867499

\section{HAL Id: hal-00867499 \\ https://hal-iogs.archives-ouvertes.fr/hal-00867499}

Submitted on 30 Sep 2013

HAL is a multi-disciplinary open access archive for the deposit and dissemination of scientific research documents, whether they are published or not. The documents may come from teaching and research institutions in France or abroad, or from public or private research centers.
L'archive ouverte pluridisciplinaire HAL, est destinée au dépôt et à la diffusion de documents scientifiques de niveau recherche, publiés ou non, émanant des établissements d'enseignement et de recherche français ou étrangers, des laboratoires publics ou privés. 


\title{
Efficient single-mode Brillouin fiber laser for low-noise optical carrier reduction of microwave signals
}

\author{
S. Norcia, S. Tonda-Goldstein, D. Dolfi, and J.-P. Huignard \\ Thales Research \& Technology, Domaine de Corbeville, 91404 Orsay cedex, France
}

R. Frey

Laboratoire Charles Fabry de l'Institut d'Optique, Centre Scientifique Paris-Sud, Bâtiment 503, 91403 Orsay cedex, France

Received April 22, 2003

\begin{abstract}
We experimentally demonstrate efficient optical carrier reduction of microwave signals with a single-mode 1.5- $\mu \mathrm{m}$ wavelength Brillouin all-fiber ring laser. Because of the tunable optical coupling, the lasing threshold of the short-length (20-m) fiber cavity is lower than $5 \mathrm{~mW}$, and high conversion efficiencies (up to 60\%) are obtained at any pump power up to $\sim 200 \mathrm{~mW}$. Using the single-mode Stokes beam as a seed for the stimulated Brillouin scattering process allows up to 40-dB optical carrier depletion with almost no added noise for an optically carried microwave signal at $6 \mathrm{GHz}$. In addition, using this resonator, we provide evidence of generation of high-spectral-purity beatnotes. () 2003 Optical Society of America
\end{abstract}

OCIS codes: $060.4370,140.3510,190.4360,190.5890$.

Since conventional optoelectronic modulators allow only limited modulation depths, optically carried radio-frequency (RF) signals decompose over low-level sidebands and a strong optical carrier, which can saturate high-speed detectors. All-fiber stimulated Brillouin scattering ${ }^{1}$ (SBS) devices can be good candidates to reach significant optical carrier reduction of microwave signals. ${ }^{2-5}$ A weakly intensity modulated optical beam is injected into a long optical fiber. The optical carrier, exceeding the SBS threshold, generates a backward-propagating Stokes wave and consequently experiences selective depletion. The proposed architectures involve long optical fibers, either in single-pass geometry or (to decrease SBS threshold) in loop configuration. In the latter case, the circulating Stokes wave is multimode, potentially resulting in additional noise that is close to carrier noise on the transmitted RF signals. Preventing this additional noise requires injection of a single-mode Stokes wave into long optical fiber. The single-mode Stokes wave is generated separately in a short optical fiber ring, whereas the modulated signal travels into the long fiber.

In this Letter we present single-mode Stokes wave generation with low-level pump power in a doubly resonant SBS laser. The SBS laser is based on an all-fiber short cavity that is simultaneously resonant for pump and Stokes waves. ${ }^{6} \quad$ As the cavity length is chosen to correspond to roughly the inverse of the SBS gain bandwidth, only one single-longitudinal-mode Stokes wave automatically oscillates in the resonator. The pump wave is resonant in the ring, drastically reducing the SBS threshold in the short-length optical fiber. Moreover, the use of a tunable optical coupler allows high conversion efficiencies at any pump power level. Using this single-mode Stokes wave as a seed for the SBS process, we clearly demonstrate reduction of phase noise on the RF signal and possible generation of spectrally pure RF signals.

The experimental setup implemented to demonstrate the efficient single-mode all-fiber Brillouin laser is described in Fig. 1. A $2 \times 2$ evanescent-mode variable optical coupler is used to close the ring cavity with a variable coupling coefficient $R$. Ports $\mathrm{C}$ and $\mathrm{D}$ of the variable optical coupler are pigtailed to both ends of a short (20-m-long) polarization-maintaining optical fiber (PMF). Part of this fiber is wound around a piezoelectric actuator (PZT) to allow control of the PMF length and, therefore, to control the phase accumulated by the pump along the ring. A single-frequency solid-state $1.5-\mu \mathrm{m}$ pump laser source (up to $200 \mathrm{~mW}$ of power and $2-\mathrm{kHz}$ spectral bandwidth) passes the optical circulator (OC) and enters the coupler in A. The pump is then divided into two beams. One beam passes the coupler and goes directly to $\mathrm{B}$, giving rise to the so-called direct beam. The other beam goes to $\mathrm{C}$ and, after passing through the PMF, enters the coupler in $\mathrm{D}$, giving rise to two beams. One beam is reinjected into the ring, and the other one (the so-called transmitted beam) is extracted via port B. The respective intensities of these beams depend on the selected coupling coefficient $R$. The values $R=1$ and $R=0$ correspond to no and total coupling, respectively. When it is detected in $\mathrm{B}$, the output pump intensity is used for feedback on the PZT actuator to control the fiber ring length to reach a resonant situation for the pump frequency. In this case, constructive interferences of the pump waves occur at port $\mathrm{C}$, and destructive interferences between the direct beam and the transmitted beam can be

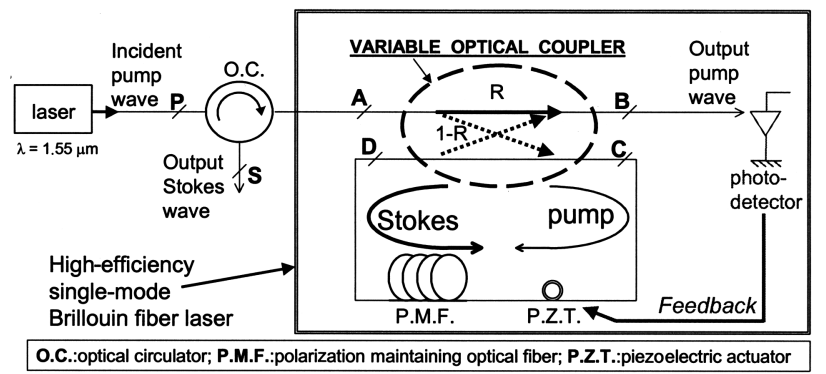

Fig. 1. Efficient single-mode Brillouin fiber laser. 
observed in the system output, B. It must be emphasized that the coupling of pump light into the cavity is maximum when no light is detected in B. For a given level of losses experienced by the pump wave in the cavity, this occurs at a given value of $R$, when the direct and transmitted beams have equal intensities.

When the SBS threshold is reached by the pump in the resonator, some of the pump energy is converted into a Stokes wave, traveling in the opposite direction in the ring. Part of the backward-propagating Stokes wave is recirculated into the ring via port $\mathrm{D}$; the other part goes out of the ring from port $\mathrm{C}$ to port $\mathrm{A}$ and is available at port $\mathrm{S}$ of the OC. When a Stokes mode falls within the $10-\mathrm{MHz}$-wide SBS gain curve, it is automatically resonant in the ring. The creation of a Stokes wave (backward propagating and downshifted in frequency from the pump's optical frequency by $\nu_{B}=11 \mathrm{GHz}$ ) induces additional losses for the pump. Maximum conversion efficiency is then obtained by adjustment of coupling coefficient $R$, taking all the losses (linear and nonlinear losses) of the pump wave in the ring into account.

To characterize the operation of this single-mode Brillouin laser, pump (in B) and Stokes (in S) intensities going out of the cavity were measured as functions of coupling coefficient $R$. For input pump powers lower than the Brillouin threshold in the resonator, the total losses of the cavity are due only to the linear absorption $\alpha$ of the PMF $\left\{\alpha \approx 2.9 \times 10^{-3} \mathrm{~m}^{-1}\right.$ and the PMF length, $L=20 \mathrm{~m}$, which corresponds to an effective length $L_{\text {eff } 1}=[1-\exp (-\alpha L)] / \alpha$, i.e., $\left.L_{\text {eff } 1} \approx 19 \mathrm{~m}\right\}$ and to the OC insertion losses $(0.5 \mathrm{~dB})$. As it is resonant for the pump frequency, this Brillouin laser allows the generation of a Stokes wave with less than $5 \mathrm{~mW}$ of input pump power. This power is $\sim 500$ times lower than the Brillouin threshold of a 20-m-long single-pass optical fiber. These $5 \mathrm{~mW}$ of pump power also correspond to the SBS threshold power of a fiber in single-pass geometry, with an effective length $L_{\text {eff } 2}=5 \mathrm{~km}$. ${ }^{1}$ An order of magnitude of the finesse of the cavity is given by the ratio $L_{\text {eff2 }} / L_{\text {eff } 1} \approx 260$. For input pump powers generating SBS, nonlinear loss is added to the cavity: While input pump power increases, maximum resonance of the pump is reached for decreasing values of $R$. This is illustrated in Fig. 2, which shows the measured output pump and Stokes intensities as functions of coupling coefficient $R$, for different input pump powers $\left(P_{\text {in }}\right)$. Indeed, maximum conversion efficiency of pump intensity into Stokes intensity occurs for a value of $R$ that depends strongly on the input pump power in the cavity. As an example, an efficiency of more than $60 \%$ is reached for $R=0.25$ with $P_{\text {in }}=190 \mathrm{~mW}$. Evidently, even higher values would be obtained with a fiber ring cavity exhibiting less loss, which appear feasible.

For Stokes powers exceeding the SBS threshold of the cavity, an additional corresponding Stokes wave is generated. We call it the 2-Stokes wave, and it propagates in the same direction as the pump. The pump and 2-Stokes powers are measured with an optical spectrum analyzer at port B. Measured values of the 2-Stokes wave (downshifted from the pump optical frequency by $2 \nu_{B}$ ) are also shown in Fig. 2 . This 2-Stokes wave disappears for input pump powers lower than $20 \mathrm{~mW}$ and is present only for high coupling coefficients. All the behaviors reported above can be described with a model based on nonlinear propagation equations.

This single-mode Brillouin laser was used for 40-dB carrier reduction of RF-modulated optical beams, as illustrated in Fig. 3. In such experiments, the Stokes wave is injected (in the reverse direction) in a long $(10-\mathrm{km})$ non-polarization-maintaining optical fiber (OF) carrying a weakly modulated RF signal. Approximately $75-\mathrm{mW}$ input power in the laser is needed to generate a $45-\mathrm{mW}$ Stokes signal at port A (see Fig. 1) and a $20-\mathrm{mW}$ Stokes signal that is available at port $\mathrm{S}$ to be injected into the long fiber (OC insertion loss is of the order of $3 \mathrm{~dB}$ ). Only $1 \mathrm{~mW}$ of optically carried $\mathrm{RF}$ signal power is required at the optical fiber input.

To possibly improve the phase-noise performance of optoelectronic links including dynamic carrier attenuation, standard characterization ${ }^{7}$ with a $R F$ synthesizer and a phase-noise bench test were performed at the Thales Air Defence facility. The experimental results are presented in Fig. 4, in which we compare the phase noise of the RF signal obtained with three different schemes of modulation depth enhancement by SBS. The figure shows the phase noise at the output end of the optical links in a 1-MHz-wide spectral range about the $\mathrm{RF}$ modulation frequency $(\sim 6 \mathrm{GHz})$. As the input modulation depth is $10 \%$, a carrier reduction of $10 \mathrm{~dB}$ is needed to reach $100 \%$ modulation depth at the link output. Reaching this carrier reduction requires injection of an $\sim 4-\mathrm{mW}$ Stokes seed. The reference level is a link with no additional phase noise on the RF signal. At frequencies higher than $1 \mathrm{kHz}$ from modulation frequency, the reference level is near $-130 \mathrm{dBc} / \mathrm{Hz}$. Seeding the fiber

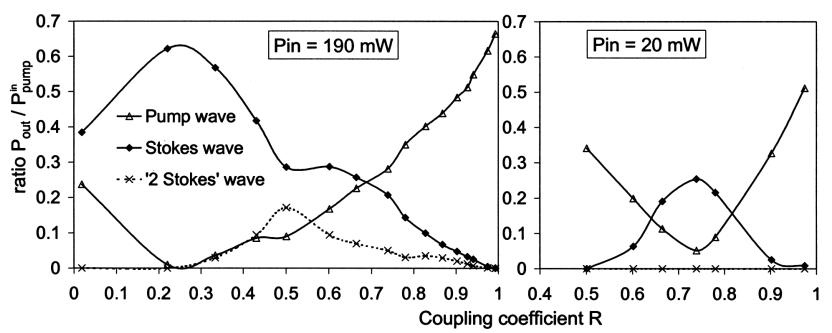

Fig. 2. Output pump, Stokes, and 2-Stokes powers as a function of coupling coefficient $R$ for two different input powers.

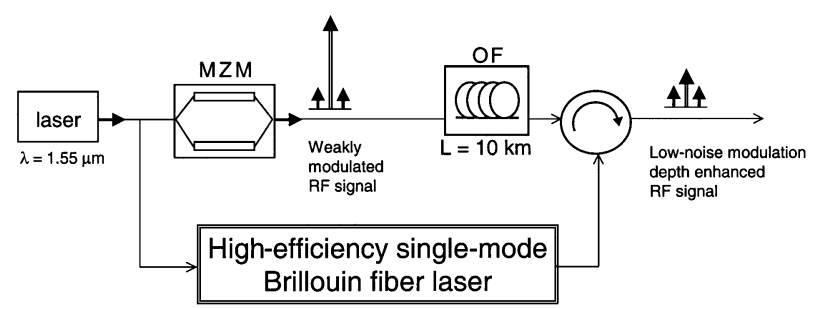

Fig. 3. Experimental setup for modulation depth enhancement of a weakly modulated RF signal. MZM, MachZehnder modulator. 


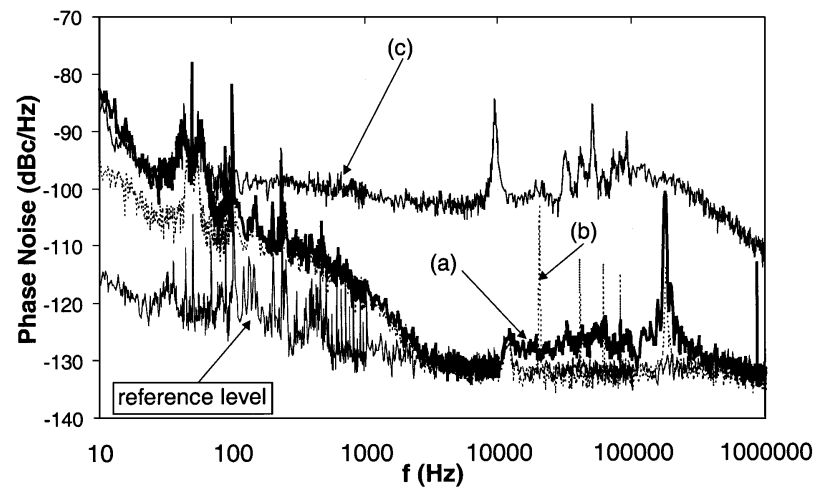

Fig. 4. Phase-noise measurements on an optically carried $\mathrm{RF}$ signal. Dynamic carrier attenuation with (a) a single-mode Stokes wave, (b) a multimode Stokes wave, (c) single-pass geometry.

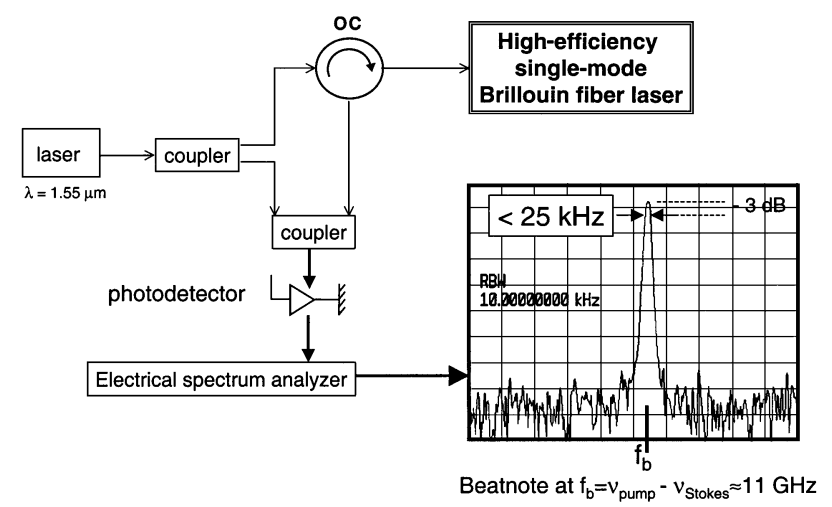

Fig. 5. Beatnote between the pump laser and the SBS laser. RBW, resolution bandwidth.

with the single-mode Stokes wave [curve (a)] permits modulation depth enhancement with almost no added phase noise. The increase of the phase-noise level at $180 \mathrm{kHz}$ is due to the relaxation oscillations of the pump laser. Injection of the multimode Stokes wave provided by a long cavity introduces periodic noise, with a period corresponding to the free spectral range of the Stokes cavity [curve (b)]. Curve (c) shows that the quality of the RF signal is drastically reduced compared with the reference level when no input Stokes seed is injected into the fiber. This case uses SBS in single-pass geometry and allows only a $3-\mathrm{dB}$ carrier reduction. These results definitely demonstrate that the single-mode Brillouin fiber laser permits very significant modulation depth enhancement with low pump power while maintaining the quality of the transmitted RF signal.

In addition, the simultaneous resonance of the pump and Stokes waves in the cavity imposes strong coherence between the pump laser and the SBS laser. In our experiments the spectral linewidth of the pump laser is measured to be only a few kilohertz wide. Such a small width can be exploited for generation of a pure RF signal through the beatnote between two optical waves in the range of the pump-to-Stokes-waves frequency offset $\left(\nu_{B}=11 \mathrm{GHz}\right)$. Using the experimental setup shown in Fig. 5, we obtain a RF beatnote at $11 \mathrm{GHz}$ with a FWHM of less than $25 \mathrm{kHz}$. Even lower values can be expected when the linewidthnarrowing properties of Brillouin lasers are used. ${ }^{8}$

In conclusion, we have demonstrated an efficient single-mode $1.5-\mu \mathrm{m}$ wavelength Brillouin all-fiber ring laser. It generates Stokes power with less than $5 \mathrm{~mW}$ of input pump power, and conversion efficiencies of up to $60 \%$ can be obtained when the tunable optical coupler of the cavity is optimized. The single-mode operation of the generated Stokes wave permits 40-dB modulation depth enhancement of optically carried $\mathrm{rf}$ signals with low input pump power and without addition of phase noise. Such a single-mode wave, downshifted in frequency by $11 \mathrm{GHz}$ from the optical pump frequency, can also be used for spectrally pure RF local oscillator generation and distribution in the receive mode of radar systems. Further investigation will be devoted to the improvement of the cavity quality factor and to the generation of tunable local oscillators with a very narrow spectral bandwidth.

We thank T. Merlet, S. Blanc (Thales Air Defence), J. Chazelas, and L. Ménager (Thales Airborne Systems) for partial support and contributions to phase-noise measurements and S. Randoux and J. Zemmouri (Centre d'Etudes et Recherches Laser et Applications, Université de Lille, France) for enlightening discussions. S. Norcia's e-mail address is stephanie.norcia@thalesgroup.com.

\section{References}

1. G. P. Agrawal, Nonlinear Fiber Optics, 3rd ed. (Academic, San Diego, Calif., 2001), Chap. 9.

2. K. J. Williams and R. D. Esman, Electron. Lett. 30, 1965 (1994).

3. A. Loayssa, D. Benito, and M. J. Garde, Opt. Lett. 25, 197 (2000).

4. S. Tonda-Goldstein, D. Dolfi, J.-P. Huignard, G. Charlet, and J. Chazelas, Electron. Lett. 36, 944 (2000).

5. S. Norcia, S. Tonda-Goldstein, D. Dolfi, and J.-P. Huignard, European Conference on Optical Communication (COM, Technical University of Denmark, Lyngby, Denmark, 2002), paper 8.2.7.

6. L. F. Stokes, M. Chodorow, and H. J. Shaw, Opt. Lett. 7, 288 (1982).

7. D. H. Harrison, M. J. Howes, and R. D. Pollard, in IEEE Microwave Theory and Techniques Society Digest 1987 (Institute of Electrical and Electronics Engineers, Piscataway, N.J., 1987), pp. 521-525.

8. A. Debut, S. Randoux, and J. Zemmouri, J. Opt. Soc. Am. B 18, 556 (2001). 